\title{
Duality in a supersymmetric gauge theory from a perturbative viewpoint
}

\author{
Thomas A. Ryttov ${ }^{1}$ and Robert Shrock ${ }^{2}$ \\ ${ }^{1}$ CP-Origins, University of Southern Denmark, Campusvej 55, Odense, Denmark \\ ${ }^{2}$ C. N. Yang Institute for Theoretical Physics and Department of Physics and Astronomy, \\ Stony Brook University, Stony Brook, New York 11794, USA
}

(Received 2 November 2017; published 30 March 2018)

\begin{abstract}
We study duality in $\mathcal{N}=1$ supersymmetric QCD in the non-Abelian Coulomb phase, order-by-order in scheme-independent series expansions. Using exact results, we show how the dimensions of various fundamental and composite chiral superfields, and the quantities $a, c, a / c$, and $b$ at superconformal fixed points of the renormalization group emerge in scheme-independent series expansions in the electric and magnetic theories. We further demonstrate that truncations of these series expansions to modest order yield very accurate approximations to these quantities and suggest possible implications for nonsupersymmetric theories.
\end{abstract}

DOI: $10.1103 /$ PhysRevD.97.065020

\section{INTRODUCTION}

Transformations that allow one to deal with a strongly coupled quantum field theory as a weakly coupled field theory in a different form have proved to be very powerful throughout the history of physics. An important example is provided by the lattice formulation of quantum chromodynamics (QCD). Although the property of asymptotic freedom made possible perturbative calculations at large Euclidean energy/momentum scales $\mu$ in the deep ultraviolet (UV), the growth of the running gauge coupling $g(\mu)$ in the infrared (IR) prevented reliable perturbative calculations at low energies. This difficulty was surmounted by Wilson's formulation of the theory on a (Euclidean) lattice [1], in which the plaquette term in the action is multiplied by the coefficient $\beta_{P}=2 N_{c} / g_{0}^{2}$, where $g_{0}$ is the bare gauge coupling. Thus, the strong coupling limit $g_{0} \rightarrow \infty$ is equivalent to $\beta_{P} \rightarrow 0$, allowing analytic strong-coupling Taylor series expansions in powers of $\beta_{P}$. From such an expansion, the area-law behavior of the Wilson loop and hence confinement in QCD were proved for strong $g_{0}$ [1]. In a different way, a duality transformation links two different regimes of a theory. For example, in the twodimensional Ising model, a duality transformation maps the high-temperature regime to the low-temperature regime and led to the calculation of the critical temperature in this model. Another realization of duality occurs in the generalization of electromagnetic theory to include Dirac monopoles.

Published by the American Physical Society under the terms of the Creative Commons Attribution 4.0 International license. Further distribution of this work must maintain attribution to the author(s) and the published article's title, journal citation, and DOI. Funded by SCOAP.
Here we will consider a theory for which duality relations have been very useful, namely an asymptotically free, vectorial, gauge theory (in $d=4$ spacetime dimensions, at zero temperature) with $\mathcal{N}=1$ supersymmetry, having a gauge group $\mathrm{SU}\left(N_{c}\right)$ and $N_{f}$ massless chiral superfields $Q^{i}$ and $\tilde{Q}_{i}, i=1, \ldots, N_{f}$, transforming in the fundamental and conjugate fundamental representations of $\mathrm{SU}\left(N_{c}\right)$, respectively. The decomposition of the chiral superfields $Q_{i}$ and $\tilde{Q}_{i}$ in terms of component fields is indicated in the standard manner as $Q_{i}=\phi_{i}+\sqrt{2} \theta \psi_{i}+$ $\theta \theta F_{i}$ and $\tilde{Q}_{i}=\tilde{\phi}_{i}+\sqrt{2} \theta \tilde{\psi}_{i}+\theta \theta \tilde{F}_{i}$, where $\theta$ is a Grassmann variable, and $\phi_{i}, \psi_{i}$, and $F_{i}$ are the scalar, fermion, and auxiliary component fields. This theory is invariant under a global symmetry group $G_{g b}=\mathrm{SU}\left(N_{f}\right) \otimes$ $\mathrm{SU}\left(N_{f}\right) \otimes \mathrm{U}(1)_{B} \otimes \mathrm{U}(1)_{R}$ symmetry, with the representations indicated in Table I. We call this theory supersymmetric quantum chromodynamics (SQCD). We denote

TABLE I. The matter content of the original (electric) and dual (magnetic) SQCD theories.

\begin{tabular}{cccccc}
\hline \hline & $\mathrm{SU}\left(N_{c}\right)$ & $\mathrm{SU}\left(N_{f}\right)$ & $\mathrm{SU}\left(N_{f}\right)$ & $\mathrm{U}(1)_{B}$ & $\mathrm{U}(1)_{R}$ \\
\hline$Q$ & $N_{c}$ & $N_{f}$ & 1 & 1 & $\frac{N_{f}-N_{c}}{N_{f}}$ \\
$\tilde{Q}$ & $\bar{N}_{c}$ & 1 & $\bar{N}_{f}$ & -1 & $\frac{N_{f}-N_{c}}{N_{f}}$ \\
\hline \hline & $\mathrm{SU}\left(N_{f}-N_{c}\right)$ & $\mathrm{SU}\left(N_{f}\right)$ & $\mathrm{SU}\left(N_{f}\right)$ & $\mathrm{U}(1)_{B}$ & $\mathrm{U}(1)_{R}$ \\
\hline$q$ & $N_{c}$ & $\bar{N}_{f}$ & 1 & $\frac{N_{c}}{N_{f}-N_{c}}$ & $\frac{N_{c}}{N_{f}}$ \\
$\tilde{q}$ & $\bar{N}_{c}$ & 1 & $N_{f}$ & $-\frac{N_{c}}{N_{f}-N_{c}}$ & $\frac{N_{c}}{N_{f}}$ \\
$\phi$ & 1 & $N_{f}$ & $\bar{N}_{f}$ & 0 & $\frac{2\left(N_{f}-N_{c}\right)}{N_{f}}$ \\
\hline \hline
\end{tabular}


$\alpha=g^{2} /(4 \pi)$. This theory has the appeal that many of its properties are well understood at a nonperturbative level [2-5]. The property of asymptotic freedom requires that $N_{f}$ be less than an upper $(u)$ bound, $N_{u}$, which, in the present theory, is $3 N_{c}$. In this range, the theory is weakly interacting in the deep UV, so one can calculate its properties perturbatively. One can then investigate how it evolves ("flows") from the deep UV to the IR limit as $\mu \rightarrow 0$. For $N_{f}$ slightly less than $3 N_{c}$, the theory evolves to an infrared fixed point (IRFP) of the renormalization group (RG) at $\alpha_{\mathrm{IR}}$, at which point it is scale-invariant and is inferred [6] to be (super)conformally invariant. If $N_{f}$ is in the interval $I:(3 / 2) N_{c}<N_{f}<3 N_{c}$, the theory flows to an IRFP in a (deconfined, chirally symmetric) non-Abelian Coulomb phase (NACP) [3]. Henceforth, we restrict our consideration to the NACP in this theory.

At this superconformal IRFP, it was conjectured in [3] that the original theory is dual to the IR limit of another $\mathcal{N}=1$ supersymmetric theory with a gauge group $\mathrm{SU}\left(\tilde{N}_{c}\right)$, where $\tilde{N}_{c}=N_{f}-N_{c}$, with matter content consisting of $N_{f}$ chiral superfields $q_{i}$ and $\tilde{q}^{i}, i=1, \ldots, N_{f}$, in the fundamental and conjugate fundamental representations of $\mathrm{SU}\left(\tilde{N}_{c}\right)$, respectively, together with a set of $N_{f}^{2}$ gaugesinglet "meson" chiral superfields $\phi_{j}^{i}, 1 \leq i, j \leq N_{f}$. The original and dual theories were called "electric" and "magnetic" in [3,4]. The dual theory also allows for a unique superpotential $W=\lambda \phi q \tilde{q}$, where $\lambda$ is the superpotential coupling. Evidence for this conjectured equivalence includes the fact that the dual theory is also invariant under the same global $G_{g b}$ symmetry, and satisfies 't Hooft anomaly matching [3-5].

The dual theory is asymptotically free for $N_{f}<3 \tilde{N}_{c}$, i.e., $N_{f}>(3 / 2) N_{c}$ and for $N_{f}$ in the interval $\tilde{I}:(3 / 2) \tilde{N}_{c}<$ $N_{f}<3 \tilde{N}_{c}$, it flows to a superconformal IRFP in the space of gauge and $\lambda$ couplings, at which the physics is equivalent to that in the original theory. The weak-coupling region in the original theory corresponds to strong coupling in the dual theory, and vice versa. This duality is well supported by nonperturbative arguments, but one gains further insight by seeing how the duality relations emerge perturbatively. However, a conventional perturbative calculation, as a series expansion in powers of the gauge coupling, encounters the difficulty that although $\alpha_{\mathrm{IR}} \rightarrow 0$ as $N_{f} \nearrow 3 N_{c}$ at the upper end of the NACP, this theory becomes strongly coupled, and hence not amenable to this type of perturbative approach, as $N_{f} \searrow(3 / 2) N_{c}$ at the lower end of the NACP. A similar comment applies to conventional perturbative expansions in the magnetic theory, which has a weak gauge coupling as $N_{f} \nearrow 3 \tilde{N}_{c}$, but is strongly coupled as $N_{f} \searrow(3 / 2) \tilde{N}_{c}$. Even in the regions of the electric and magnetic theories where they are weakly coupled, perturbative expansions in powers of gauge couplings are schemedependent.
Here we surmount this difficulty and present, for the first time, a scheme-independent perturbative understanding of the duality in the non-Abelian Coulomb phase of SQCD. We utilize the fact that $\alpha_{\mathrm{IR}} \rightarrow 0$ as $N_{f} \nearrow 3 N_{c}$, so that one can alternatively express physical quantities at an IRFP in the NACP as series in powers of the manifestly schemeindependent quantity [7-9]

$$
\Delta_{f}=3 N_{c}-N_{f}
$$

The expansion parameter in the dual theory is

$$
\tilde{\Delta}_{f}=3 \tilde{N}_{c}-N_{f}=2 N_{f}-3 N_{c} .
$$

We will study dimensions of various (gauge-invariant) chiral superfield operators and of certain quantities $a, c$, $a / c$, and $b$ characterizing RG flows, in both the original and dual theories, as expansions in both $\Delta_{f}$ and $\tilde{\Delta}_{f}$, and will show how various relations emerge order-by-order in these expansions. We address and answer an important question, namely how accurate are finite truncations of these series expansions as approximations to the exact results for the various scaling dimensions. Clearly, the series expansions in powers of $\Delta_{f}$ are most accurate at the upper end of the NACP, while the series expansions in powers of $\tilde{\Delta}_{f}$ are most accurate at the lower end of the NACP. From our analysis, we will show that a combination of finite-order expansions in these two dual expansion parameters $\Delta_{f}$ and $\tilde{\Delta}_{f}$ yields quite accurate approximations to physical quantities throughout the entire NACP. Our work thus demonstrates how perturbative calculations in the well-chosen scheme-independent expansion parameters $\Delta_{f}$ and $\tilde{\Delta}_{f}$ can provide insight into results based on abstract nonperturbative methods. Our present results extend our previous studies of anomalous dimensions in $\mathcal{N}=1$ supersymmetric gauge theories $[10,11]$ in showing how well a combination of perturbative expansions in $\Delta_{f}$ and $\tilde{\Delta}_{f}$, reproduce exactly known results for the anomalous dimensions of fundamental and composite chiral superfields, as well as quantities such as $a$ and $c$. For this purpose, we make use of the powerful electric-magnetic duality in this theory. Just as exact results in SQCD [2-5] have provided new insights into QCD with many fermions (e.g., the NACP), so also, our quantitative demonstration of the accuracy of finite truncations of these schemeindependent series suggest that corresponding finite-order scheme-independent series [9] in the NACP of nonsupersymmetric theories might be similarly accurate.

\section{BACKGROUND AND CALCULATIONAL METHODS}

In this section we review some background and methods relevant for our calculations. The series expansion of $\beta$ in powers of $\alpha$ is 


$$
\beta=-2 \alpha \sum_{\ell=1}^{\infty} b_{\ell}\left(\frac{\alpha}{4 \pi}\right)^{\ell},
$$

where $b_{\ell}$ is the $\ell$-loop coefficient. The first two coefficients in the expansion (2.1) are scheme-independent, while higher-order coefficients are scheme-dependent. For this theory, the first two coefficients are [12]

$$
b_{1}=3 N_{c}-N_{f}
$$

and [13]

$$
b_{2}=2\left[3 N_{c}^{2}-\left(2 N_{c}-N_{c}^{-1}\right) N_{f}\right] .
$$

For $N_{f}$ slightly less than $3 N_{c}$, the two-loop beta function has a (scheme-independent) IR zero at

$$
\alpha_{\mathrm{IR}, 2 \ell}=\frac{2 \pi\left(3 N_{c}-N_{f}\right)}{\left(2 N_{c}-N_{c}^{-1}\right) N_{f}-3 N_{c}^{2}} .
$$

If $N_{f}$ is only slightly less than $3 N_{c}$, then this IRFP occurs at weak coupling. Indeed, in the limit $N_{c} \rightarrow \infty, N_{f} \rightarrow \infty$ with the ratio $r=N_{f} / N_{c}$ fixed, the IRFP in the scaled coupling $\xi=\alpha N_{c}$ (which is finite in this limit) occurs at

$$
\xi_{\mathrm{IR}, 2 \ell}=\frac{2 \pi(3-r)}{2 r-3}
$$

and becomes arbitrarily weak as $r \nearrow 3$.

One can express a (gauge-invariant) physical quantity $\mathcal{P}$ at this IRFP as a power series in the coupling,

$$
\mathcal{P}=\sum_{n=0}^{\infty} c_{\mathcal{P}, n} \alpha_{\mathrm{IR}}^{n}
$$

where the $c_{\mathcal{P}, n}$ are $n$-loop coefficients, and it is understood implicitly here that $\alpha_{\mathrm{IR}}$ is the value of $\alpha$ at the IRFP, calculated to the $n$-loop order, to match the $n$-loop order of the expansion of $\mathcal{P}$. One important example of a physical quantity is the (full) scaling dimension $D_{\mathcal{O}}$ of a physical operator $\mathcal{O}$ at an IRFP, which, in general, differs from its free-field value, due to interactions. We write

$$
D_{\mathcal{O}}=D_{\mathcal{O}, \text { free }}-\gamma_{\mathcal{O}}
$$

where $D_{\mathcal{O} \text {,free }}$ is the free-field scaling dimension of $\mathcal{O}$ and $\gamma_{\mathcal{O}}$ is the anomalous dimension at the IRFP. In general (not assuming that the theory is at an IRFP) the anomalous dimension can be expanded as a conventional series in powers of the coupling as

$$
\gamma_{\mathcal{O}}=\sum_{\ell=1}^{\infty} c_{\mathcal{O}, \ell} \alpha^{\ell}
$$

At an IRFP, one can calculate this to a given $n$-loop order by computing the $n$-loop value of $\alpha$ and substituting it in Eq. (2.8). This was done for $\mathcal{O}=\bar{\psi} \psi=\sum_{i=1}^{N_{f}} \bar{\psi}_{i} \psi_{i}$ in [10], $[14,15]$, and effects of scheme dependence were studied in [16]. Connections between the $\overline{\mathrm{DR}}$ [17] and NSVZ [2] schemes have been discussed in [18].

As a physical quantity, $\mathcal{P}$ cannot depend on the scheme used in the conventional calculation as the power series (2.9). However, this property is not maintained beyond the lowest orders in Eq. (2.9). It is therefore of great value to devise a complementary approach in which one expresses a physical quantity evaluated at the IRFP $\alpha=\alpha_{\mathrm{IR}}$ as an expansion in powers of a variable such that, at every order in this expansion, the result is scheme-independent. The key property that makes this possible is the fact that both $\alpha_{\mathrm{IR}}$ and $\Delta_{f}$ vanish as $N_{f}$ approaches $3 N_{c}$ from below. Because of this, one can recast the conventional series (2.9) as a power series in the manifestly scheme-independent variable $\Delta_{f}$. By the same logic, one can recast a series for $\mathcal{P}$ in the dual, magnetic theory as a series in powers of $\tilde{\Delta}_{f}$. This successfully accomplishes three goals: (i) achieving series for the quantity $\mathcal{P}$ at the IRFP in the non-Abelian Coulomb phase in both the electric and magnetic theories that are manifestly scheme-independent, (ii) having a series in the magnetic theory involving a small expansion parameter $\tilde{\Delta}_{f}$ that can be used accurately in the region near the lower end of the NACP where the expansion parameter $\Delta_{f}$ in the electric theory is getting large, and hence (iii) having accurate perturbative expansions starting from both ends and extending throughout the entirety of the non-Abelian Coulomb phase.

For a (gauge-invariant) quantity $\mathcal{P}$ at the superconformal IRFP, we write the expansions of $\mathcal{P}$ in powers of $\Delta_{f}$ and $\tilde{\Delta}_{f}$ as

$$
\mathcal{P}=\sum_{n=0}^{\infty} p_{n} \Delta_{f}^{n}=\sum_{n=0}^{\infty} \tilde{p}_{n} \tilde{\Delta}_{f}^{n} .
$$

In the first sum in Eq. (2.9), one takes $N_{c}$ as fixed and computes $\mathcal{P}$ as a function of the variable $\Delta_{f}$, or equivalently, $N_{f}$. In the second sum, for the dual theory, one takes $\tilde{N}_{c}=N_{f}-N_{c}$ as fixed; while varying $N_{f}$, one can keep $\tilde{N}_{c}$ fixed by formally varying $N_{c}$ oppositely to $N_{f}$. The $n$ th-order coefficients have a definite relation to terms in conventional perturbative expansions.

At an IRFP, $\gamma_{\mathcal{O}}$ can also be expanded in a schemeindependent series as

$$
\gamma_{\mathcal{O}}=\sum_{n=1}^{\infty} \kappa_{\mathcal{O}, n} \Delta_{f}^{n} .
$$

The coefficient $\kappa_{\mathcal{O}, n}$ depends on the terms in the beta function of the theory in Eq. (2.1) up to loop order $\ell=$ $n+1$ and on the terms in the conventional expansion of 
$\gamma_{\mathcal{O}}$ in powers of $\alpha$ in Eq. (2.8) up to loop order $\ell=n$, inclusive, but does not receive contributions from higherloop terms. This is a very powerful observation which can be used in two different ways. First, it allows for a calculation of any physical quantity in a manifestly scheme-independent way, order-by-order, even if one only knows the gauge coupling beta function and $D_{\mathcal{O}}$ to some finite loop order. Second, if, on the other hand, one knows an exact expression for a quantity $\mathcal{P}$ at the IRFP, then one can calculate its expansions in powers of $\Delta_{f}$ and $\tilde{\Delta}_{f}$ in Eq. (2.9) exactly, to all orders, without doing any explicit loop computations.

In the analysis in the present work, we will use the second method, since the exact expressions for the relevant operator dimensions are known in this $\mathcal{N}=1$ supersymmetric gauge theory. To make our discussion self-contained, we will also review the explicit method of calculating the coefficients $\kappa_{\mathcal{O}, n}$ from knowledge of the coefficients $b_{n}$ and $c_{\mathcal{P}, n}$ in conventional series in powers of $\alpha$. (For further details of this method, we refer the reader to our previous papers in [9].) For definiteness, we consider the case of the gauge-invariant quadratic chiral superfield operator product $\tilde{Q} Q$ (where a sum over flavor indices is understood) and focus on the anomalous dimension $\gamma_{\tilde{\psi} \psi, \text { IR }}$ of the associated fermionic bilinear $\tilde{\psi} \psi$ at the IRFP, where, as defined above, $\psi_{i}$ and $\tilde{\psi}_{i}$ are the fermion field components in the chiral superfields $Q_{i}$ and $\tilde{Q}_{i}$. This anomalous dimension is the analogue, in this supersymmetric theory, of the quantity $\gamma_{\bar{\psi} \psi, \text {,IR }}$ in nonsupersymmetric gauge theories that we have calculated schemeindependent $\Delta_{f}$ expansions for in previous work [9]. The conventional expansion for $\gamma_{\tilde{\psi} \psi, \text { IR }}$ in the electric theory has the form (2.9) with $c_{\tilde{\psi} \psi, n} \equiv c_{n}$. For the scheme-independent expansion in the electric theory we write

$$
\gamma_{\tilde{\psi} \psi, \mathrm{IR}}=\sum_{j=1}^{\infty} \kappa_{j} \Delta_{f}^{j} .
$$

To calculate $\kappa_{j}$, one begins by writing $a_{\mathrm{IR}}=\alpha_{\mathrm{IR}} /(4 \pi)$ as a series expansion in $\Delta_{f}$ :

$$
a_{\mathrm{IR}}=\sum_{j=1}^{\infty} a_{j} \Delta_{f}^{j} .
$$

For an IR zero of $\beta$, we have

$$
\sum_{\ell=1}^{\infty} b_{\ell} a^{\ell}=0
$$

Next, one expands the coefficients $b_{\ell}$ in Taylor series around $N_{f}=N_{u}$, i.e., $\Delta_{f}=0$. Since each $b_{\ell}$ is a finite polynomial in $N_{f}$, its Taylor series around $\Delta_{f}=0$ truncates at a maximal degree denoted $r_{\max }(\ell)$. One then substitutes the resulting expansions for $b_{\ell}$ and the $\Delta_{f}$ expansion for $a_{\mathrm{IR}}$, Eq. (2.12), in Eq. (2.13), yielding

$$
\begin{aligned}
\sum_{\ell=1}^{\infty} b_{\ell} a^{\ell} & =0=\sum_{\ell=1}^{\infty}\left[\left(\sum_{r=0}^{r_{\max }(\ell)} b_{\ell}^{(r)} \Delta_{f}^{r}\right)\left(\sum_{j=1}^{\infty} a_{j} \Delta_{f}^{j}\right)^{\ell}\right] \\
& =\sum_{n=1}^{\infty} k_{n} \Delta_{f}^{n} .
\end{aligned}
$$

Since the sum in the last line of Eq. (2.14) is zero for all $\Delta_{f}$, each coefficient $k_{n}$ must be zero. This yields a set of linear equations that one can solve for the $a_{n}$ in terms of the Taylor series coefficients of $b_{\ell}$ in the expansion about $\Delta_{f}=0$. Next, one inserts these expressions for the $a_{n}$ in the $\Delta_{f}$ expansion for $a_{\mathrm{IR}}$. Then, one carries out similar Taylor series expansions of the $c_{n}$ around $\Delta_{f}=0$ and substitutes these, together with the expansion (2.12) of the $a_{\mathrm{IR}}$, in the series $\gamma_{\tilde{\psi} \psi, \mathrm{IR}}=\sum_{\ell=0}^{\infty} c_{\ell} a_{\mathrm{IR}}^{\ell}$. This finally yields the coefficients $\kappa_{j}$ in Eq. (2.11). This, then, is the procedure to obtain the coefficients in the scheme-independent series expansion of $\gamma_{\bar{\psi} \psi, \text { IR }}$ in powers of $\Delta_{f}$. The same type of procedure is used to obtain the coefficients in the scheme-independent series expansions of other physical quantities at the IRFP.

\section{RESULTS FOR MESON AND BARYON OPERATORS}

In the original (electric) theory, gauge-singlet composite chiral superfields include the meson-type operators $M^{i}{ }_{j}=Q^{i} \tilde{Q}_{j}$, and baryon- and antibaryon-type operators

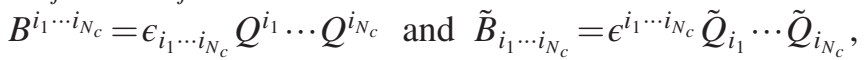
respectively. Similarly, in the dual (magnetic) theory, in addition to $\phi_{j}^{i}$, one has the dual baryon and antibaryon operators $b_{i_{1} \cdots i_{\tilde{N}_{c}}}=\epsilon^{i_{1} \cdots i_{\tilde{N}_{c}}} q_{i_{1}} \cdots q_{i_{\tilde{N}_{c}}}$ and $\tilde{b}^{i_{1} \cdots i_{\tilde{N}_{c}}}=\epsilon_{i_{1} \cdots i_{\tilde{N}_{c}}} \tilde{q}^{i_{1} \cdots}$ $\tilde{q}^{i \tilde{N}_{c}}$. Duality dictates that the meson operators are matched in the electric and magnetic theories, and similarly for the baryon operators and the antibaryon operators. Hence, the meson operators in the electric and magnetic theories must have the same dimensions, and similarly for the baryon and antibaryon operators.

In a superconformal theory, the dimension $D_{\mathcal{O}}$ of a gauge-invariant chiral superfield operator $\mathcal{O}$ is related to the $R$-charge, $R_{\mathcal{O}}$, of the operator via $D_{\mathcal{O}}=(3 / 2) R_{\mathcal{O}}$. This implies, in particular, that the scaling dimension of the (composite) electric and (fundamental) magnetic mesons is

$$
D_{M}=D_{\phi} \equiv D=\frac{3\left(N_{f}-N_{c}\right)}{N_{f}}=\frac{3 \tilde{N}_{c}}{N_{f}} .
$$

Note that $D_{M \text {,free }}=2$ while $D_{\phi \text {,free }}=1$. We want to understand how Eq. (3.1) emerges order-by-order in the schemeindependent expansions in the original and dual theories. To calculate the series expansion of $D$ in powers of $\Delta_{f}$, we substitute $N_{f}=3 N_{c}-\Delta_{f}$ in Eq. (3.1) and expand, obtaining 


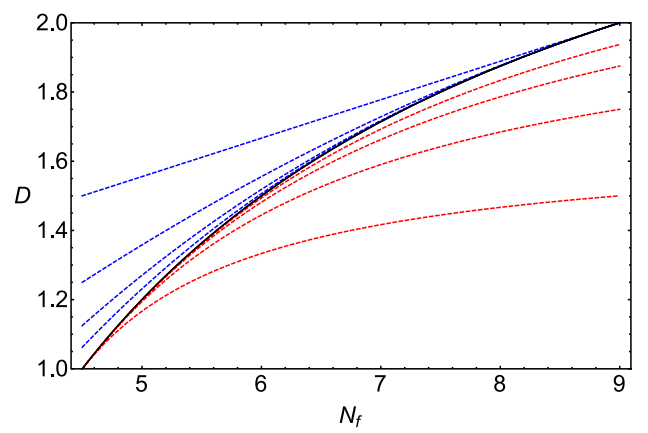

FIG. 1. The scaling dimension $D$ of the meson chiral superfield, as computed to $O\left(\Delta_{f}^{s}\right)$ in the original (electric) theory (blue dashed curves) and to $O\left(\tilde{\Delta}_{f}^{s}\right)$ in the dual (magnetic) theory (red dashed curves), together with the exact $D$ (black solid curve), for the illustrative case $N_{c}=3$. From top to bottom for the blue curves and from bottom to top for the red curves, these depict $D_{s}$ with $s=1, \ldots, 4$.

$$
D=2-\sum_{n=1}^{\infty}\left(\frac{\Delta_{f}}{3 N_{c}}\right)^{n}
$$

Equivalently, in the dual theory, substituting $N_{f}=3 \tilde{N}_{c}-\tilde{\Delta}_{f}$ in Eq. (3.1) and expanding, we find

$$
D=1+\sum_{n=1}^{\infty}\left(\frac{\tilde{\Delta}_{f}}{3 \tilde{N}_{c}}\right)^{n}
$$

These series have respective radii of convergence $\left|\Delta_{f}\right|=$ $3 N_{c}$ and $\left|\tilde{\Delta}_{f}\right|=3 \tilde{N}_{c}$, and hence converge throughout the entire NACP, since the maximal values of $\Delta_{f}$ and $\tilde{\Delta}_{f}$ in the NACP are $(3 / 2) N_{c}$ and $(3 / 2) \tilde{N}_{c}$. The truncations of the series (3.2) and (3.3) to order $n=s$ are denoted $D_{s}$.

In Fig. 1 we plot the values of $D$ calculated from (3.2) to $O\left(\Delta_{f}^{s}\right)$, i.e., $D_{s}$, in the electric theory and $D_{s}$ calculated from (3.3) in the magnetic theory for $1 \leq s \leq 4$, together with the exact result, for the illustrative case, $N_{c}=3$. The maximal order $s=4$ is chosen to be the same as the highest order in our SI expansions in nonsupersymmetric theories, to facilitate accuracy comparisons.

Because the coefficients of $\Delta_{f}^{n}$ and $\tilde{\Delta}_{f}^{n}$ in the expansions (3.2) and (3.3) are positive, several monotonicity properties follow (for $N_{f}$ in the NACP): (i) for fixed $s, D_{s}$ is a monotonically increasing function of $N_{f}$; (ii) for fixed $N_{f}$, $D_{s}$, as calculated in the electric theory, decreases monotonically with $s$; and (iii) $D_{s}$, as calculated in the magnetic theory, increases monotonically with $s$. As is evident from Fig. 1 , the respective fractional accuracies of the $\Delta_{f}$ and $\tilde{\Delta}_{f}$ series expansions in (3.2) and (3.3) are highest near the upper and lower ends of the NACP, respectively. Thus, by combining these two perturbative calculations, we achieve an excellent approximation to the exact expression (3.1) throughout all of the NACP, even with a modest value of the

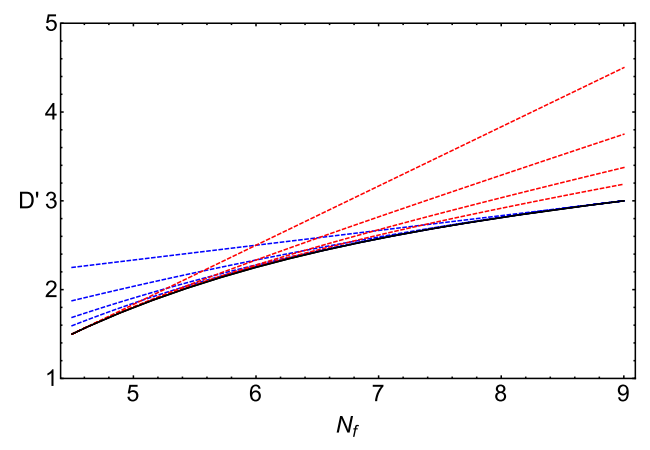

FIG. 2. The scaling dimension $D^{\prime}$ of the baryon operators, as computed in the electric theory (blue dashed curves) and magnetic theory (red dashed curves), together with the exact result (black solid curve), for the illustrative value, $N_{c}=3$. From top to bottom for both the blue and red curves, these depict $D_{s}^{\prime}$ with $s=1, \ldots, 4$.

truncation order, $s$ such as $s=4$. This makes use of the full power of the duality, since it allows one to treat the strongcoupling regime in the original theory via a perturbative calculation in the weak-coupling regime of the dual theory, and vice versa.

We next consider the baryon-type operators. In both the electric and magnetic theories, their scaling dimensions have to agree, and are

$$
D_{B}=D_{b} \equiv D^{\prime}=\frac{3 N_{c}\left(N_{f}-N_{c}\right)}{2 N_{f}}=\frac{3 N_{c} \tilde{N}_{c}}{2 N_{f}}
$$

As with the mesons, we want to understand how this expression for $D^{\prime}$ emerges order-by-order in perturbation theory, as calculated in both the original and dual theories. In the original (electric) theory, we find

$$
D^{\prime}=N_{c}-\frac{N_{c}}{2} \sum_{n=1}^{\infty}\left(\frac{\Delta_{f}}{3 N_{c}}\right)^{n}
$$

while in the dual (magnetic) theory we find

$$
D^{\prime}=\tilde{N}_{c}-\frac{\tilde{N}_{c}}{2} \sum_{n=1}^{\infty}\left(\frac{\tilde{\Delta}_{f}}{3 \tilde{N}_{c}}\right)^{n}
$$

As before, these series converge throughout the entire NACP. The truncations of these series to order $n=s$ are denoted $D_{s}^{\prime}$.

In Fig. 2 we plot the values of $D^{\prime}$ calculated from (3.5) to $O\left(\Delta_{f}^{s}\right)$ in the electric theory and from (3.6) to $O\left(\tilde{\Delta}_{f}^{s}\right)$ in the magnetic theory, for $1 \leq s \leq 4$, together with the exact result. Because the coefficients of $\Delta_{f}^{n}$ and $\tilde{\Delta}_{f}^{n}$ in the expansions (3.5) and (3.6) are positive, several monotonicity properties follow for the calculations in both the electric and magnetic theories (for $N_{f}$ in the NACP): (i) for fixed $s, D_{s}^{\prime}$ is a monotonically increasing function of $N_{f}$; 
and (ii) for fixed $N_{f}, D_{s}^{\prime}$ is a monotonically decreasing function of $s$. The same comments about respective fractional accuracies of the $\Delta_{f}$ and $\tilde{\Delta}_{f}$ calculations made for Fig. 1 hold here, so that again, by combining these two perturbative calculations, we obtain an excellent approximation to the exact expression (3.4) throughout all of the NACP even with a modest truncation order, $s$, such as $s=4$.

Corresponding to the global symmetry groups in $G_{g b}$, there are conserved currents. We will focus on the conserved current $J_{\mu}^{(B)}$ associated with the $\mathrm{U}(1)_{B}$ baryon number symmetry. The two-point correlation function for this current (in flat space) is

$$
\left\langle J_{\mu}^{(B)}(x) J_{\nu}^{(B)}(0)\right\rangle=\frac{b}{(4 \pi)^{2}}\left(g_{\mu \nu} \partial^{2}-\partial_{\mu} \partial_{\nu}\right) \frac{1}{x^{4}} .
$$

Here $b$ is a function of the couplings of the theory and changes from $b_{\mathrm{UV}}$ to $b_{\mathrm{IR}}$ along the RG flow. For SQCD, $b_{\mathrm{UV}}$ is given by its respective free-field values, $b_{\mathrm{UV}}=$ $2 N_{f} / N_{c}$ and $b_{\mathrm{UV}}=2 N_{f} / \tilde{N}_{c}$ in the electric and magnetic theories, while $b_{\mathrm{IR}}=6$ at the IRFP in the non-Abelian Coulomb phase in both of these theories [19]. Hence, calculating $b_{\mathrm{UV}}-b_{\mathrm{IR}}$, we have, in the electric theory,

$$
b_{\mathrm{UV}}-b_{\mathrm{IR}}=-\frac{2 \Delta_{f}}{N_{c}},
$$

and, in the magnetic theory,

$$
b_{\mathrm{UV}}-b_{\mathrm{IR}}=-\frac{2 \tilde{\Delta}_{f}}{\tilde{N}_{c}} .
$$

These results show that higher-order contributions to $b_{\mathrm{IR}}$ in powers of $\Delta_{f}$, and equivalently in powers of $\tilde{\Delta}_{f}$, vanish. This again shows the value of the scheme-independent series expansion method, since the zero coefficients of the respective $\Delta_{f}^{n}$ and $\tilde{\Delta}_{f}^{n}$ terms with $n \geq 2$ in Eqs. (3.8) and (3.9) involve complicated cancellations when computed via conventional (scheme-dependent) series expansions in powers of couplings.

\section{ANALYSIS OF $a$ AND $c$}

The trace of the energy-momentum tensor in four spacetime dimensions, in the presence of a curved background metric $g_{\mu \nu}$, is [20]

$$
T_{\mu}^{\mu}=\frac{1}{(4 \pi)^{2}}\left(c W_{\mu \nu \rho \sigma} W^{\mu \nu \rho \sigma}-a E_{4}\right)
$$

where $W_{\mu \nu \rho \sigma}$ is the Weyl tensor and $E_{4}$ is the Euler density. In $d=2$, it was proved that $c$ decreases monotonically along an RG flow [21], but this monotonicity does not hold in $d=4$. The quantity $a$ encodes important information about the flow of a quantum field theory between two RG fixed points and satisfies the inequality that $a_{\mathrm{UV}}-a_{\mathrm{IR}}>0$ (called the $a$ theorem) [19,22-26]. This is in accord with the Wilsonian notion of thinning of degrees of freedom along an RG flow [27]. For an asymptotically free theory, $a_{\mathrm{UV}}$ is given by the (massless) free-field content of the theory [20]: $a_{\mathrm{UV}}=(1 / 48)\left(9 N_{v}+N_{\chi}\right)$, where here $N_{v}$ and $N_{\chi}$ denote the numbers of vector and chiral superfields, respectively [20]. In the original (electric) theory, $N_{v}=$ $N_{c}^{2}-1$ and $N_{\chi}=2 N_{c} N_{f}$, while in the dual (magnetic) theory, $N_{v}=\tilde{N}_{c}^{2}-1$ and $N_{\chi}=2 \tilde{N}_{c} N_{f}+N_{f}^{2}$. The duality at the superconformal IRFP dictates that the value of $a_{\text {IR }}$ must be identical in the electric and magnetic theories; it is [19] $a_{\mathrm{IR}}=(3 / 16)\left[2 N_{c}^{2}-1-3\left(N_{c}^{4} / N_{f}^{2}\right)\right]$. Note that this result for $a_{\mathrm{IR}}$ does not contain any factors $\sim e^{- \text {const. } / \alpha_{\mathrm{IR}}}$ that are generic manifestations of instanton effects. Calculating a series expansion for $a_{\mathrm{IR}}$ in the electric theory, in powers of $\Delta_{f}$, we obtain

$$
a_{\mathrm{IR}}=a_{\mathrm{UV}}-\frac{1}{144} \sum_{n=2}^{\infty} \frac{(n+1) \Delta_{f}^{n}}{\left(3 N_{c}\right)^{n-2}} .
$$

Equation (4.2) shows how the $a$ theorem is satisfied at each order in powers of $\Delta_{f}$. In the magnetic theory, we find

$$
a_{\mathrm{IR}}=a_{\mathrm{UV}}-\frac{1}{144}\left[21 \tilde{\Delta}_{f}^{2}+\sum_{n=3}^{\infty} \frac{(n-11) \tilde{\Delta}_{f}^{n}}{\left(3 \tilde{N}_{c}\right)^{n-2}}\right] .
$$

Here again, the $a$ theorem is satisfied at each order in powers of $\tilde{\Delta}_{f}^{n}$; in this case, the result follows because the leading-order term in the square brackets, $21 \tilde{\Delta}_{f}^{2}$, dominates over higher-order terms $\propto \tilde{\Delta}_{f}^{n}$ with $3 \leq n \leq 10$ with opposite sign.

In Fig. 3 we plot $a_{\mathrm{IR}}$ as computed to first through fourth order in $\Delta_{f}$ in the electric theory and in $\tilde{\Delta}_{f}$ in the magnetic theory, together with the exact result, for the illustrative case $N_{c}=3$.

Although the coefficient $c$ in Eq. (4.1) does not obey a monotonicity relation along RG flows or an inequality on the sign of $c_{\mathrm{UV}}-c_{\mathrm{IR}}$, it is also of interest to calculate scheme-independent series expansions for this difference. For the (asymptotically free) $\mathcal{N}=1$ supersymmetric gauge theory considered here, $c_{\mathrm{UV}}=(1 / 24)\left(3 N_{v}+N_{\chi}\right)$, where, as above, $N_{v}$ and $N_{\chi}$ denote the numbers of (massless) vector and chiral superfields [20]. The duality at the superconformal IRFP implies that the value of $c_{\text {IR }}$ must be identical in the electric and magnetic theories; it is [19] $c_{\mathrm{IR}}=(1 / 16)\left[7 N_{c}^{2}-2-\left(9 N_{c}^{4} / N_{f}^{2}\right)\right]$. Calculating a series expansion for $c_{\mathrm{IR}}$ in the electric theory, in powers of $\Delta_{f}$, we obtain

$$
c_{\mathrm{IR}}=c_{\mathrm{UV}}+\frac{N_{c}}{48}\left(2 \Delta_{f}-\sum_{n=2}^{\infty} \frac{(n+1) \Delta_{f}^{n}}{\left(3 N_{c}\right)^{n-1}}\right) .
$$




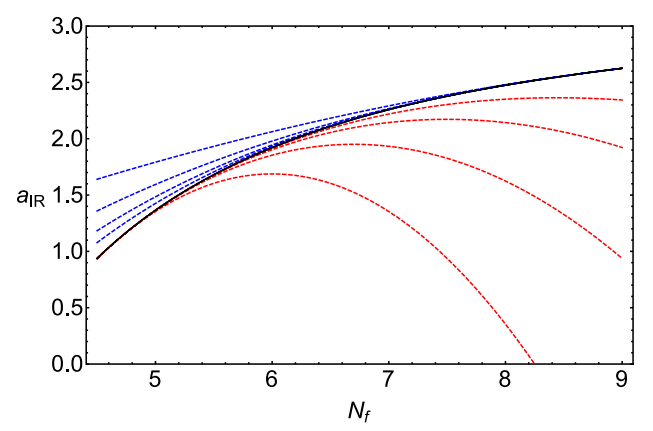

FIG. 3. The quantity $a_{\mathrm{IR}}$, as computed in the electric theory (blue dashed curves) and magnetic theory (red dashed curves), together with the exact result (black solid curve), for the illustrative case $N_{c}=3$. From top to bottom, the blue curves are for $a_{\mathrm{IR}}$ calculated to $O\left(\Delta_{f}^{s}\right)$ with $s=1, \ldots, 4$, and from bottom to top, the red curves are for $a_{\mathrm{IR}}$ calculated to $O\left(\tilde{\Delta}_{f}^{s}\right)$ with $s=1, \ldots, 4$.

In the magnetic theory, we find

$c_{\mathrm{IR}}=c_{\mathrm{UV}}-\frac{\tilde{N}_{c}}{48}\left(4 \tilde{\Delta}_{f}+\frac{5 \tilde{\Delta}_{f}^{2}}{\tilde{N}_{c}}+\sum_{n=3}^{\infty} \frac{(n-11) \tilde{\Delta}_{f}^{n}}{\left(3 \tilde{N}_{c}\right)^{n-1}}\right)$.

In Fig. 4 we plot $c_{\mathrm{IR}}$ as computed to first through fourth order in $\Delta_{f}$ in the electric theory and in $\tilde{\Delta}_{f}$ in the magnetic theory, together with the exact result (black solid curve), for the illustrative case $N_{c}=3$. The curves in Fig. 4 show the same result as we demonstrated for operator dimensions and for $a_{\mathrm{IR}}$, namely that by combining $\Delta_{f}$ and $\tilde{\Delta}_{f}$ series expansions, one can get very good approximations to exact nonperturbative expressions, even for modest truncation orders.

In a superconformal field theory, the ratio $a / c$ is restricted, by the positivity of energy flux, to lie in the interval $1 / 2 \leq a / c \leq 3 / 2$ [28-30]. (This ratio $a / c$ saturates its upper bound of $3 / 2$ if the superconformal theory contains only vector superfields, while it saturates its lower bound of $1 / 2$ if this theory contains only chiral superfields.) We have calculated $\Delta_{f}$ and $\tilde{\Delta}_{f}$ series expansions for $a_{\mathrm{IR}} / c_{\mathrm{IR}}$ and, as with $a_{\mathrm{IR}}$ and $c_{\mathrm{IR}}$, we have found that that a combination of modest-length series in these two variables gives a very good approximation to the exact result.

\section{CONCLUSIONS}

In conclusion, we have shown, for the first time, how exact relations for dimensions of chiral superfields and for the quantities $a, c, a / c$, and $b$ in the non-Abelian

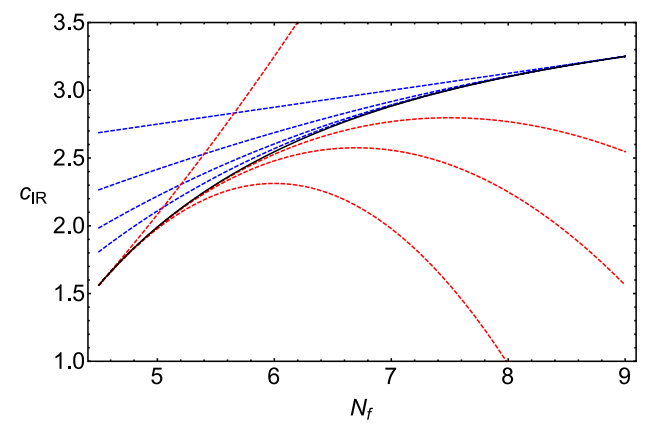

FIG. 4. The central charge $c_{\mathrm{IR}}$, as computed in the electric theory (blue dashed curves) and magnetic theory (red dashed curves), together with the exact result (black solid curve), for the illustrative case $N_{c}=3$. For the electric theory, from top to bottom, the curves refer to the calculation of $c_{\mathrm{IR}}$ to $O\left(\Delta_{f}^{s}\right)$ with $s=1, \ldots, 4$, while for the magnetic theory, from bottom to top, the curves refer to the calculation of $c_{\mathrm{IR}}$ to $O\left(\tilde{\Delta}_{f}^{s}\right)$ with $s=1, \ldots, 4$.

Coulomb phase of SQCD emerge order-by-order in scheme-independent perturbative series expansions, as calculated in the original (electric) theory in powers of $\Delta_{f}$ and in the dual magnetic theory in powers of $\tilde{\Delta}_{f}$. Our series expansions have a fundamental advantage over conventional series expansions of these quantities in powers of $\alpha_{\mathrm{IR}}$, namely that our expansions are schemeindependent, while conventional expansions in powers of $\alpha_{\mathrm{IR}}$ are scheme-dependent. We have addressed and answered the question of how well finite-order truncations of these scheme-independent series expansions approximate exact expressions. We have demonstrated that a combination of truncated $\Delta_{f}$ and $\tilde{\Delta}_{f}$ series expansions of modest order yield quite accurate approximations to exact results for these quantities throughout the entire nonAbelian Coulomb phase. Although these results do not apply directly to nonsupersymmetric theories, they encourage one in the conjecture that corresponding finite-order series [9] in the NACP of a nonsupersymmetric gauge theory might be similarly accurate.

\section{ACKNOWLEDGMENTS}

The research of T.A.R. and R.S. was supported in part by the Danish National Research Foundation Grant DNRF90 to $\mathrm{CP}^{3}$-Origins at SDU and by the U.S. National Science Foundation Grant NSF-PHY-16-1620628, respectively. 
[1] K. G. Wilson, Phys. Rev. D 10, 2445 (1974).

[2] V. A. Novikov, M. A. Shifman, A. I. Vainshtein, and V. I. Zakharov, Phys. Lett. 166B, 329 (1986).

[3] N. Seiberg, Nucl. Phys. B435, 129 (1995).

[4] K. A. Intriligator and N. Seiberg, Nucl. Phys. B, Proc. Suppl. 45, 1 (1996).

[5] M. A. Shifman, Prog. Part. Nucl. Phys. 39, 1 (1997).

[6] For a recent review, see Y. Nakayama, Phys. Rep. 569, 1 (2015).

[7] T. Banks and A. Zaks, Nucl. Phys. B196, 189 (1982).

[8] E. Gardi and M. Karliner, Nucl. Phys. B529, 383 (1998); E. Gardi and G. Grunberg, J. High Energy Phys. 03 (1999) 024.

[9] T. A. Ryttov, Phys. Rev. Lett. 117, 071601 (2016); T. A. Ryttov and R. Shrock, Phys. Rev. D 94, 105014 (2016); 94, 125005 (2016); 95, 085012 (2017); 95, 105004 (2017); 96, 105015 (2017); 97, 025004 (2018); 97, 016020 (2018).

[10] T. A. Ryttov and R. Shrock, Phys. Rev. D 85, 076009 (2012); R. Shrock, Phys. Rev. D 87, 105005 (2013); 87, 116007 (2013); 91, 125039 (2015); G. Choi and R. Shrock, Phys. Rev. D 93, 065013 (2016).

[11] T. A. Ryttov and R. Shrock, Phys. Rev. D 96, 105018 (2017).

[12] D. R. T. Jones, Nucl. Phys. B87, 127 (1975).

[13] M. Machacek and M. Vaughn, Nucl. Phys. B222, 83 (1983); A. J. Parkes and P. C. West, Phys. Lett. 138B, 99 (1984); D. R. T. Jones and L. Mezincescu, Phys. Lett. 136B, 242 (1984).

[14] T. A. Ryttov and R. Shrock, Phys. Rev. D 83, 056011 (2011); C. Pica and F. Sannino, Phys. Rev. D 83, 035013 (2011).

[15] T. A. Ryttov and R. Shrock, Phys. Rev. D 94, 105015 (2016).

[16] T. A. Ryttov and R. Shrock, Phys. Rev. D 86, 065032 (2012); 86, 085005 (2012); R. Shrock, Phys. Rev. D 88, 036003 (2013); T. A. Ryttov, Phys. Rev. D 89, 056001 (2014); 89, 016013 (2014); R. Shrock, Phys. Rev. D 90,
045011 (2014); T. A. Ryttov, Phys. Rev. D 90, 056007 (2014); G. Choi and R. Shrock, Phys. Rev. D 90, 125029 (2014); J. A. Gracey and R. M. Simms, Phys. Rev. D 91, 085037 (2015); G. Choi and R. Shrock, Phys. Rev. D 94, 065038 (2016).

[17] W. Siegel, Phys. Lett. 84B, 193 (1979); 94B, 37 (1980); D. M. Capper, D. R. T. Jones, and P. van Nieuwenhuizen, Nucl. Phys. B167, 479 (1980); W. Stöckinger, J. High Energy Phys. 03 (2005) 076.

[18] I. Jack, D. R. T. Jones, and C. G. North, Nucl. Phys. B486, 479 (1997); I. Jack, D. R. T. Jones, and A. Pickering, Phys. Lett. B 435, 61 (1998); A. L. Kataev and K. V. Stepanyantz, Theor. Math. Phys. 181, 1531 (2014).

[19] D. Anselmi, D. Z. Freedman, M. T. Grisaru, and A. A. Johansen, Nucl. Phys. B526, 543 (1998).

[20] M. J. Duff, Nucl. Phys. B125, 334 (1977); arXiv:hep-th/ 9308075 and references therein.

[21] A. B. Zamolodchikov, Pis'ma Zh. Eksp. Teor. Fiz. 43, 565 (1986) [JETP Lett. 43, 730 (1986)].

[22] J. Cardy, Phys. Lett. B 215, 749 (1988).

[23] H. Osborne, Phys. Lett. B 222, 97 (1989); I. Jack and H. Osborn, Nucl. Phys. B343, 647 (1990).

[24] D. Anselmi, J. Erlich, D. Z. Freedman, and A. A. Johansen, Phys. Rev. D 57, 7570 (1998).

[25] D. Z. Freedman and H. Osborn, Phys. Lett. B 432, 353 (1998).

[26] Z. Komargodski and A. Schwimmer, J. High Energy Phys. 12 (2011) 099.

[27] For a thermally motivated conjecture on degrees of freedom in RG flows, see T. Appelquist, A. G. Cohen, and M. Schmaltz, Phys. Rev. D 60, 045003 (1999); T. Appelquest et al., Phys. Lett. B 459, 235 (1999).

[28] D. M. Hofman and J. Maldacena, J. High Energy Phys. 05 (2008) 012.

[29] D. M. Hofman et al., J. High Energy Phys. 06 (2016) 111.

[30] C. Cordova, J. Maldacena, and G. J. Turiaci, J. High Energy Phys. 11 (2017) 032. 